\title{
QUOTIENT RINGS OF SEMIPRIME RINGS WITH BOUNDED INDEX
}

\author{
by JOHN HANNAH
}

(Received 8 July, 1980)

We say that a ring $R$ has bounded index if there is a positive integer $n$ such that $a^{n}=0$ for each nilpotent element $a$ of $R$. If $n$ is the least such integer we say $R$ has index $n$. For example, any semiprime right Goldie ring has bounded index, and so does any semiprime ring satisfying a polynomial identity [10, Theorem 10.8.2]. This paper is mainly concerned with the maximal (right) quotient ring $Q$ of a semiprime ring $R$ with bounded index. Several special cases of this situation have already received attention in the literature. If $R$ satisfies a polynomial identity [1], or if every nonzero right ideal of $R$ contains a nonzero idempotent [18] then it is known that $Q$ is a finite direct product of matrix rings over strongly regular self-injective rings, the size of the matrices being bounded by the index of $R$. On the other hand if $R$ is reduced (that is, has index 1 ) then $Q$ is a direct product of a strongly regular self-injective ring and a biregular right self-injective ring of type III ([2] and [15]; the terminology is explained in [6]). We prove the following generalization of these results (see Theorems 9 and 11).

THEOREM. Let $R$ be a semiprime ring of index $n$ and let $Q$ be its maximal right quotient ring. Then $Q=Q_{1} \oplus Q_{3}$ where $Q_{3}$ is a biregular right self-injective ring of type III, and where $Q_{1}$ is a finite direct product of matrix rings over strongly regular self-injective rings, the size of the matrices being at most $n \times n$.

We also show that the natural attempt to generalize this theorem to right nonsingular rings with bounded index breaks down quite badly. We give an example where $Q$ is itself type III but not biregular, and show (Proposition 15) that every regular right self-injective ring of type $I$ is the maximal quotient ring of a ring with index at most 2 . The only part of the theorem which remains intact is that $Q$ still cannot have a direct factor of type II (Theorem 17).

None of the techniques used in the special cases mentioned above seems to work for general semiprime rings with bounded index, so we must start from scratch. In section 1 we develop some basic properties of rings with bounded index (most of these are probably well-known but $I$ have been unable to find a suitable reference for them). For our purposes the main point to emerge is that if $R$ is semiprime with bounded index then $R$ is (right) nonsingular. This implies that the maximal right quotient ring $Q$ of $R$ is regular and right self-injective, and means that we have a decomposition of $Q$ into a direct product of type I, II and III rings (as described in [6] and [7]). In section 2 we show how properties of the maximal quotient ring can induce nilpotent elements in the original ring (Lemma 7). We use this to simplify the decomposition of $Q$, and thus obtain the results mentioned earlier.

In what follows all rings are associative but they need not have an identity element. A

Glasgow Math. J. 23 (1982) 53-64. 
ring is semiprime if it has no nonzero nilpotent ideals. We denote the right annihilator of a subset $X$ of a ring $R$ by $r_{R}(X)$ or simply $r(X)$. The left annihilator of $X$ is denoted by $l_{R}(X)$ or $l(X)$. For general background on ring theory we refer to [10]. The basic results about maximal quotient rings which we use are proved in [3]. For results about regular self-injective rings we refer to [6], [7] and [8]. Any unexplained terminology or notation may be found in [6].

ACKNOWLEDGEMENT. I would like to thank Jim Richardson for helping me clarify some of the ideas in this paper.

1. General structure. We begin with a lemma which, though quite elementary, is the key idea in most of the following results. It is based on a trick used by Kaplansky in [11, Theorem 2.3] (although the exact connexion may not be apparent until we use it in Lemma 7).

LEMMA 1. Let $R$ be a ring with index at most $n$. If $X_{1}, X_{2}, \ldots, X_{n}$ are subsets of $R$ such that $X_{i} X_{j}=0$ whenever $i \geq j$ then $X_{1} X_{2} \ldots X_{n}=0$.

Proof. If $x_{i} \in X_{i}$ for $1 \leq i \leq n$ consider $z=x_{1}+x_{2}+\ldots+x_{n}$. By hypothesis $z^{n}=$ $x_{1} x_{2} \ldots x_{n}$ and $z^{n+1}=0$. As $R$ has index at most $n$ we get $x_{1} x_{2} \ldots x_{n}=0$ and the result follows.

The property in Lemma 1 does not in general characterize rings with bounded index: any commutative ring has the property. However it is equivalent if $R$ is semiprime. In fact we have:

Proposirion 2. Let $R$ be a semiprime ring and $n$ a positive integer. The following statements are equivalent.

(a) $R$ has index at most $n$.

(b) If $X_{1}, X_{2}, \ldots, X_{n} \subseteq R$ such that $X_{i} X_{j}=0$ whenever $i \geq j$ then $X_{1} X_{2} \ldots X_{n}=0$.

(c) If $X \subseteq R$ then $r\left(X^{n}\right)=r\left(X^{n+1}\right)$.

(d) For each $x \in R$ we have $r\left(x^{n}\right)=r\left(x^{n+1}\right)$.

Proof. Lemma 1 gives (a) $\Rightarrow$ (b) and (c) $\Rightarrow$ (d) is trivial. If $R$ satisfies (d) and $x \in R$ then $r\left(x^{n}\right)=r\left(x^{k}\right)$ for all $k \geq n$. Hence if $x$ is nilpotent, $r\left(x^{n}\right)=R$. As $R$ is semiprime it follows that $x^{n}=0$ and (a) is true. Now suppose $R$ satisfies. (b). We imitate the proof of [16, Lemma 8]. Suppose $X \subseteq R$ and for each $i$ let $X_{i}=r\left(X^{i}\right) X^{i}$. If $i \geq j$ then $X_{i} X_{i}=0$ and so our hypothesis gives $0=X_{1} X_{2} \ldots X_{n}=[r(X)]\left[X r\left(X^{2}\right)\right] \ldots\left[X^{n-1} r\left(X^{n}\right)\right] X^{n}$. As each term in square brackets contains $X^{n} r\left(X^{n+1}\right)$ we get $\left[X^{n} r\left(X^{n+1}\right)\right]^{n+1}=0$. Since $R$ is semiprime the right ideal $X^{n} r\left(X^{n+1}\right)$ is zero. Hence $r\left(X^{n+1}\right) \subseteq r\left(X^{n}\right)$ and (c) follows.

REMARKs. (1) As statement (a) is left-right symmetric we could of course have used left, rather than right, annihilators in (c) and (d).

(2) If $R$ is not semiprime the equivalence of (a) and (c) breaks down. To see this consider the algebra $R$ constructed by Nagata in $[13, \S 5]$. Then $R$ has bounded index but it is easy to check that $r\left(R^{n}\right) \neq r\left(R^{n+1}\right)$ for each $n$. I do not know whether (a) and (d) are still equivalent without the semiprimeness of $R$. 
(3) Proposition 2 generalizes Rowen's Lemma 8 in [16] which says that (c) holds whenever $R$ is a semiprime ring for which $x_{1} x_{2} \ldots x_{n}$ is an almost pivotal monomial. I do not know whether all the rings described in Proposition 2 must have an almost pivotal monomial. However it should be noted that all the properties which Rowen deduces for semiprime rings with an almost pivotal monomial are also possessed by semiprime rings of bounded index.

We can also use Lemma 1 to prove the following result. See [10, Theorem 10.8.2] for the corresponding result for P.I. algebras.

Proposition 3. Suppose $R$ has index at most $n$ and let $N$ be the sum of the nilpotent ideals of $R$. If $S$ is a nil subring of $R$ then $S^{n} \subseteq N$.

Proof. Suppose first that $S$ is nilpotent. Let $k$ be the least integer such that $S^{k} R$ is nilpotent and suppose that $k>n$. The ideal $I=R S^{k} R$ is nilpotent and so the ring $R / I$ has index at most $n$. If we write $X_{i}=S^{k-i} R S^{i}$ for $i=1, \ldots, n$ then for each $i \geq j$ we have $X_{i} X_{i}=S^{k-i} R S^{k+i-j} R S^{j} \subseteq I$. Applying Lemma 1 in the ring $R / I$ thus gives $X_{1} X_{2} \ldots X_{n} \subseteq I$. Thus $\left(S^{k-1} R S\right)\left(S^{k-2} R S^{2}\right) \ldots\left(S^{k-n} R S^{n}\right) \subseteq I$ and so $\left(S^{k-1} R\right)^{n+1} \subseteq I$. This implies that $S^{k-1} R$ is nilpotent which is a contradiction. Hence $S^{n} R$ is nilpotent and $S^{n}+S^{n} R+R S^{n}+$ $R S^{n} R$ is a nilpotent ideal of $R$ containing $S^{n}$. Thus $S^{n} \subseteq N$.

Now let $S$ be any nil subring of $R$. By [12] $S$ is locally nilpotent. Hence if $s_{1}, \ldots, s_{n} \in S$ the subring $T$ generated by $s_{1}, \ldots, s_{n}$ satisfies $T^{n} \subseteq N$. In particular $s_{1} s_{2} \ldots s_{n} \in N$ and so $S^{n} \subseteq N$ as required.

Remark. Let $R$ be a nil ring of index $n$ and let $N$ be the sum of the nilpotent ideals of $R$. The proposition shows that $R / N$ is nilpotent. However $R \neq N$ is general. An example may be constructed in the same way as for the corresponding question for P.I. algebras $[10$, p. 233]. Thus let $A$ be any nil ring of bounded index which is not nilpotent (as, for instance, in $[13, \S 5])$. Let $B$ be the ring obtained by adjoining an identity to $A$ and let $R$ be the ring of all matrices of the form $\left(\begin{array}{cc}w & x \\ y & z\end{array}\right)$ where $w, y, z \in A$ and $x \in B$. Then $R$ is nil of bounded index (see [17, p. 138 Corollary]) but is not a sum of nilpotent ideals since the ideal $R\left(\begin{array}{cc}0 & 1 \\ 0 & 0\end{array}\right) R$ contains the subring $\left\{\left(\begin{array}{cc}a & 0 \\ 0 & 0\end{array}\right): a \in A^{2}\right\}$ and so cannot be nilpotent.

As a consequence of Propositions 2 and 3 we have the following.

Proposition 4. Let $R$ be a semiprime ring of index $n$.

(a) If $S$ is a nil subring of $R$ then $S^{n}=0$.

(b) $R$ has no nonzero nil right (or left) ideals.

(c) $R$ is right and left nonsingular.

Proof. (a) and (b) follow immediately from Proposition 3 and then (c) follows exactly as in [16, Theorem 9(ii)].

Proposition 4 generalizes [16, Theorem 9] which in turn generalizes the corresponding results for semiprime P.I. rings (due to Amitsur [10, Theorem 10.8.2] and Fisher [4]). Part (c) also generalizes the well known fact that a reduced ring is nonsingular [2, p. 139].

It follows from Proposition 4(c) that any semiprime ring of bounded index has a 
maximal right (left) quotient ring which is regular and right (left, respectively) selfinjective (see [3, p. 69]). We shall study these maximal quotient rings in more detail in the next section.

Our next result shows that Proposition 2 can be considerably strengthened if the ring involved is prime. This result generalizes [16, Proposition 3] and the well known fact that any prime P.I. ring satisfies the ascending chain condition on right (or left) annihilator ideals (see [14, p. 180]).

Propostrion 5. Suppose $R$ is a prime ring and $n$ is a positive integer. The following are equivalent.

(a) $R$ has index at most $n$.

(b) Every chain of right (left) annihilators in $R$ has at most $n$ proper inclusions.

Proof. (b) $\Rightarrow$ (a) follows as in Proposition 2 so suppose (a) is true. Once again we follow Rowen's argument [16, Proposition 3]. Let

$$
A_{1} \subset A_{2} \subset \ldots \subset A_{1}
$$

be a chain of right annihilator ideals. We suppose that $A_{1} \neq 0$ and $A_{t} \neq R$ and show that $t<n$. For each $i$ let $X_{i}=A_{i} l\left(A_{i}\right)$ so that for $i \geq j$ we have $X_{i} X_{j}=0$.

If $t \geq n$ then Lemma 1 gives $X_{1} X_{2} \ldots X_{n}=0$ and so

$$
A_{1}\left[l\left(A_{1}\right) A_{2}\right] \ldots\left[l\left(A_{n-1}\right) A_{n}\right] l\left(A_{n}\right)=0 .
$$

Now if some $l\left(A_{i-1}\right) A_{i}=0$ we have $A_{i} \subseteq r\left(l\left(A_{i-1}\right)\right)=A_{i-1}$ since $A_{i-1}$ is a right annihilator ideal. Since this contradicts $A_{i-1} \subset A_{i}$ we see that each $l\left(A_{i-1}\right) A_{i}$ is a nonzero left ideal $R$. But $A_{1}$ is a nonzero right ideal of $R$ and $l\left(A_{n}\right)$ is a nonzero left ideal (as $A_{n} \neq R$ and is a right annihilator). Thus $t \geq n$ contradicts the primeness of $R$. Hence $t<n$ and the result follows.

This strengthening of Proposition 2 is not true for general semiprime rings, as may be seen by considering the case where $R$ is an infinite direct product of fields.

Recall that a ring $R$ is said to be right strongly prime if every nonzero $a \in R$ has a right insulator, that is, a finite subset $X$ of $R$ such that $r(a X)=0$ (see [9]). If each of these right insulators can be chosen with $m$ elements (or fewer) we say that $R$ is bounded right strongly prime. If $m$ is the least such integer we say, for short, that $R$ is right $\operatorname{SP}(m)$. Left $S P(m)$ rings are defined similarly.

Corollary 6. Let $R$ be a prime ring of index $n$. Then $R$ is right $S P(m)$ for some $m \leq n$.

Proof. This now follows as in [9, Proposition I.1] (the proof there still works if $R$ does not have an identity element).

Remarks. (1) It is quite possible, in Corollary 6, that $m<n$ and that $m$ takes a different value when $R$ is viewed as a left strongly prime ring. Indeed suppose $S$ is a left Ore domain which does not satisfy the right Ore condition (see, for example, [5, p. 219]) and let $R=M_{n}(S)$, the $n \times n$ matrix ring over $S$, where $n>1$. Then $R$ is a prime ring of index exactly $n$ ( $R$ cannot contain nilpotent elements of greater index since it is a subring 
of $M_{n}(D)$ where $D$ is the division ring of left fractions of $S$ ). By Corollary $6 R$ is right $S P(m)$ for some $m \leq n$. But if $m>1$ then $R$ is a right Goldie ring (by [8, Theorem 2.3]) which is impossible as $S$ is not a right Ore domain. Hence $R$ must be right $S P(1)$. On the other hand $R$ is left $S P(n)$ by [9, Proposition I.2].

(2) Let $R$ be as in Corollary 6. If $R$ is $S P(m)$ where $m>1$ we have just remarked that $R$ is a right Goldie ring. It follows from the Faith-Utumi theorem (see [3, p. 91]) that $R$ contains the ring of $m \times m$ matrices over some right Ore domain and that $m=n$. It would be interesting to know whether all prime rings of index $n$ must contain (and/or be contained in) the ring of $n \times n$ matrices over some domain.

2. Maximal quotient rings. Let $R$ be a semiprime ring with bounded index of nilpotence. We have seen in Proposition 4 that $R$ is right nonsingular and so has a regular right self-injective maximal right quotient ring $Q$. It follows from [6, Theorem 10.13] that $Q$ is a direct product $Q_{1} \oplus Q_{2} \oplus Q_{3}$ where the rings $Q_{1}, Q_{2}, Q_{3}$ are of types I. II. III (respectively). In this section we study the structure of these rings $Q_{1}, Q_{2}, Q_{3}$ to see what effect the bounded index of $R$ has on them. We begin with a couple of technical lemmas, the first of which gives us the basic construction we shall be using.

Lemma 7. Let $R$ be a right nonsingular ring of index $n$ and let $Q$ be the maximal right quotient ring of $R$. Suppose $a_{1}, a_{2}, \ldots, a_{n+1} \in R$ such that $0 \neq a_{n+1} \in Q a_{1} R$ and for each $i>1$ we have $a_{n+1} \in Q a_{i}^{2} R$ and $a_{i} \in r\left(a_{1}, a_{2}, \ldots, a_{i-1}\right)$. Then at least one of the right ideals $a_{i} R$ contains a nonzero nilpotent right ideal of $R$.

Proof. Suppose that no $a_{i} R$ contains a nonzero nilpotent right ideal of $R$. For each $i=1,2, \ldots, n$ let $X_{i}=a_{i+1} R a_{i}$ so that if $i \leq j$ we have $X_{i} X_{j}=0$ (since $a_{i} a_{j+1}=0$ ). Since $R$ has index $n$ we can use the mirror image of Lemma 1 to deduce that $0=$ $X_{n} X_{n-1} \ldots X_{2} X_{1}=a_{n+1} R a_{n}^{2} R \ldots R a_{2}^{2} R a_{1}$. Hence $a_{n}^{2} R \ldots a_{2}^{2} R a_{1} R a_{n+1} R$ is a nilpotent right ideal of $R$ inside $a_{n} R$. By hypothesis $a_{n}^{2} R \ldots a_{2}^{2} R a_{1} R a_{n+1} R=0$ and so, as $a_{n+1} \in$ $Q a_{n}^{2} R$, we get $a_{n+1} R a_{n-1}^{2} R \ldots a_{2}^{2} R a_{1} R a_{n+1} R=0$.

We continue like this, moving the left-hand factor $a_{n+1} R$ to the right-hand end, and replacing the $a_{i}^{2} R$ factor which has been exposed by another $a_{n+1} R$ factor. When the factor $a_{2}^{2} R$ has finally been replaced we have the conclusion $a_{n+1} R a_{1} R\left(a_{n+1} R\right)^{n-1}=0$. The right ideal $a_{1} R\left(a_{n+1} R\right)^{n}$ is then nilpotent and so zero. But we then get the contradiction $\left(a_{n+1} R\right)^{n+1} \subseteq Q a_{1} R\left(a_{n+1} R\right)^{n}=0$. Hence at least one $a_{i} R$ must contain a nonzero nilpotent right ideal.

LEMMA 8. Let $R$ be a ring with a regular right quotient ring $Q$. If $X$ is a right ideal of $Q$ and $0 \neq q \in Q$ such that $q Q \leq X$ then there is a nonzero $x \in X \cap Q q R \cap R$ such that $x Q \leq q Q$.

Proof. Suppose $a \in X$ with $a Q \cong q Q$. As $Q$ is regular $a=y q z$ for some $y, z \in Q$ (see, for instance, the proof of [7, Lemma 11.1(b)]). As $Q$ is a right quotient ring of $R$ the right ideal $L=\{r \in R: z r \in R\}$ is essential in $R$. Since $Q_{R}$ is nonsingular [3, p. 59 Proposition 4] we have $a L \neq 0$ and so $a L \cap R \neq 0$. As $a L \cap R \subseteq X \cap Q q R \cap R$ and as any $x \in a L \cap R$ satisfies $x Q \subseteq a Q \cong q Q$ the proof is complete. 
Our first main result in this section describes the subrings $Q_{1}$ and $Q_{2}$ mentioned above. A special case of this result (that when $R$ has index 1 ) was proved in [2, Théorème $2.2]$.

THEOREM 9. Let $R$ be a semiprime ring of index $n$. Let $Q$ be its maximal right quotient ring and suppose that $Q=Q_{1} \oplus Q_{2} \oplus Q_{3}$ is the decomposition of $Q$ into its type I, II and III parts (respectively). Then $Q_{2}=0$ and $Q_{1}$ is a finite direct product of rings of the form $M_{k}(D)$ where $k \leq n$ and $D$ is a strongly regular self-injective ring.

Proof. We proceed in two steps. Firstly suppose that $0 \neq a \in R$ such that $a Q$ is directly finite. We show that $Q$ contains at most $n$ independent copies of $a Q$.

Suppose on the contrary that $(n+1) a Q \leqslant Q$. We shall construct a sequence of elements $a_{1}, a_{2}, \ldots, a_{n+1}$ satisfying the hypotheses of Lemma 7 . Since $R$ is semiprime Lemma 7 will then give the desired contradiction. Set $a_{1}=a$ and suppose we have found $a_{1}, a_{2}, \ldots, a_{\mathrm{k}} \in R$ such that

(i) $a_{i} \in r\left(a_{1}, a_{2}, \ldots, a_{i-1}\right)$ whenever $1<i \leq k$,

(ii) $a_{i} Q \leq a Q$ for $1 \leq i \leq k$,

(iii) $a_{k} \in Q a_{1} R \cap Q a_{i}^{2} R$ for $1<i \leq k$ and $a_{k}^{2} \neq 0$.

If $k \leq n$ we proceed as follows. Let $f: Q \rightarrow a_{1} Q \oplus \ldots \oplus a_{k} Q$ be the homomorphism $f(q)=\left(a_{1} q, \ldots, a_{k} q\right)$ so that $\operatorname{ker} f=r\left(a_{1}, \ldots, a_{k}\right)$. As $Q$ is regular $f(Q)$ is projective and so $Q \cong \operatorname{ker} f \oplus f(Q) \leqslant r\left(a_{1}, \ldots, a_{k}\right) \oplus k(a Q)$ by (ii) of the induction hypothesis. Since $k+1 \leq n+1$ we thus have $(k+1) a Q \leq r\left(a_{1}, \ldots, a_{k}\right) \oplus k(a Q)$. Since $a Q$ is directly finite we can apply the cancellation result $\left[6\right.$, Corollary 9.19] to deduce that $a Q \leq r\left(a_{1}, \ldots, a_{k}\right)$. In particular $a_{k}^{2} Q \leq r\left(a_{1}, \ldots, a_{k}\right)$. By Lemma 8 there is a nonzero $a_{k+1} \in$ $r\left(a_{1}, \ldots, a_{k}\right) \cap Q a_{k}^{2} R \cap R$ such that $a_{k+1} Q \leqslant a_{k}^{2} Q \leqslant a Q$. Furthermore, by Proposition 4(b), the right ideal $a_{k+1} R$ is not nil and so we may assume that $a_{k+1}^{2} \neq 0$. This completes the induction. Thus we have elements $a_{1}, a_{2}, \ldots, a_{n+1}$ which contradict Lemma 7 . Hence we cannot have $(n+1) a Q \leq Q$.

Now $Q$, being regular and right self-injective, can be written as a direct product $Q_{1} \oplus Q_{2} \oplus Q_{3}$ where the rings $Q_{1}, Q_{2}, Q_{3}$ are of types I, II, III respectively (by [6, Theorem 10.13]). If $Q_{2} \neq 0$ then $Q$ contains a nonzero directly finite idempotent $e$ such that $e Q$ contains no nonzero abelian idempotents of $Q$. By [7, Corollary 5.8] there is a right ideal $A$ of $Q$ such that $e Q \cong(n+1) A$. Choosing a nonzero $a \in A \cap R$ then yields $(n+1) a Q \leq Q$ and $a Q$ is still directly finite. As this contradicts the first part of the proof we must have $Q_{2}=0$.

Similarly, since every nonzero right ideal of $Q_{1}$ contains a nonzero abelian idempotent and since any abelian idempotent is directly finite, the first part of the proof shows that $Q_{1}$ does not contain any families of $n+1$ nonzero independent pairwise isomorphic right ideals. By [6, Theorem 7.2] $Q_{1}$ has index at most $n$. By [6, Theorem 7.20] it follows that $Q_{1}$ is a finite direct product of matrix rings over strongly regular self-injective rings. Since the index of $Q_{1}$ is at most $n$ the size of the matrix rings appearing in this direct product cannot be greater than $n \times n$ and so the result is proved.

Remarks. (1) The final part of the proof showed that if $R$ is semiprime of index $n$ 
then (retaining the above notation) $Q_{1}$ has index at most $n$. In particular if the type III part of $Q$ is zero (so that $Q=Q_{1}$ ) then $R$ and $Q$ have the same index.

(2) Under stronger hypotheses it is possible to show that the $Q_{3}$ in Theorem 9 is also zero. This is the case, for example, if $R$ is semiprime and satisfies a polynomial identity [1, Introduction and Theorem 3.1]. Another such case occurs when every nonzero right ideal of the ring $R$ in Theorem 9 contains a nonzero idempotent (see [18, Theorem 5 and (2) on page 13]). However $Q_{3} \neq 0$ in general. For instance if $R$ is a domain not satisfying the right Ore condition then $Q$ itself is of type III [2, Corollaire 2.3].

(3) Of course the left-hand version of Theorem 9 yields a similar decomposition for $Q^{\prime}$ the maximal left quotient ring of $R$. Indeed, in the cases treated by Armendariz and Steinberg [1] and Utumi [18] (see the previous remark) we actually have $Q=Q^{\prime}$. However in general the two decompositions need not even be of the same type. For example, if $R$ is a left but not a right Ore domain then $Q^{\prime}$ is a division ring (and so of type $I_{1}$ ) while $Q$ is of type III.

Although $Q_{3}$ need not be zero in Theorem 9 it does have a simpler structure than the general run of type III rings. Before proving this we need another lemma (which has been adapted from [15, Lemme 3.5]).

LEMMA 10. Let $Q$ be a regular right self-injective ring such that if $0 \neq q \in Q$ then there is a central idempotent $v \neq 0$ such that $v Q \leq q Q$. Then $Q$ is biregular.

Proof. Suppose $0 \neq q \in Q$. We want a central idempotent $u \in Q$ such that $Q q Q=u Q$. Choose a maximal family $\left\{v_{i} Q: i \in I\right\}$ of independent ideals where each $v_{i}$ is a nonzero central idempotent of $Q$ such that $v_{i} Q \leq q Q$. Let $u$ be the central idempotent $\bigvee_{i \in I} v_{i}$ (see [6, Proposition 9.9]).

Since each $v_{i}$ is a central idempotent the embedding $v_{i} Q \leqslant q Q$ is actually an embedding $v_{i} Q \leq v_{i} q Q$. As the ideals $v_{i} Q$ are independent we have an embedding $\sum_{i \in I} v_{i} Q \subseteq \sum_{i \in I} v_{i} q Q \subseteq q Q$. Since $u Q$ is simply the injective envelope of $\sum_{i \in I} v_{i} Q$ [6, Proposition 9.9] we thus have $u Q \leqslant q Q$ (since $q Q$ is injective). Since $Q$ is regular it follows that $u \in Q q Q$ [6, Corollary 2.23]. If $Q q Q \neq u Q$ we would have $(1-u) q \neq 0$. But then our hypothesis gives a nonzero central idempotent $w \in Q$ such that $w Q \leqslant(1-u) q Q$. Since this implies that $w Q \leq q Q$ and that $w \in(1-u) Q$ we would then have a strictly larger family $\left\{v_{i} Q: i \in I\right\} \cup\{w Q\}$ of independent nonzero ideals each of which is embeddable in $q Q$. This contradiction shows that $Q q Q=u Q$ as required.

THEOREM 11. Let $R$ be a semiprime ring of index $n$. Let $Q$ be its maximal right quotient ring and let $Q_{3}$ be the type III part of $Q$. Then $Q_{3}$, and so $Q$, is a biregular ring.

Proof. In view of Theorem 9 and [15, Théorème 2.3] it is enough to show that $Q_{3}$ is biregular. Let $0 \neq q \in Q_{3}$ and choose a nonzero $a_{1} \in q Q \cap R$. In view of Lemma 10 it is enough to find a nonzero central idempotent $v \in Q$ such that $v Q \leqslant a_{1} Q$. Once again we construct a sequence $a_{1}, a_{2}, \ldots$ as in Lemma 7 .

If $r_{Q}\left(a_{1}\right) Q a_{1}=0$ then $r_{Q}\left(a_{1}\right) \subseteq l\left(Q a_{1} Q\right)=r\left(Q a_{1} Q\right)=(1-v) Q$ where $v \neq 0$ is the 
central cover of $a_{1} Q[6$, p. 130]. Hence there is a natural epimorphism

$$
a_{1} Q \cong Q / r_{Q}\left(a_{1}\right) \rightarrow Q /(1-v) Q \cong v Q .
$$

Since $v Q$ is projective this implies $v Q \leq a_{1} Q$ and we are finished. So we suppose that $r_{Q}\left(a_{1}\right) Q a_{1} \neq 0$. Then $r_{\mathrm{Q}}\left(a_{1}\right) \cap Q a_{1} Q \neq 0$ and so $r_{\mathrm{Q}}\left(a_{1}\right) \cap Q a_{1} R \cap R \neq 0$ (as in the proof of Lemma 8). By Proposition 4(b) we can choose $a_{2} \in r_{Q}\left(a_{1}\right) \cap Q a_{1} R \cap R$ such that $a_{2}^{2} \neq 0$. Since $a_{2} \in Q a_{1} Q$ we have $a_{2} Q \leq k\left(a_{1} Q\right)$ for some integer $k$ [6, Corollary 2.23] and since $a_{1} Q$ is of type III this implies $a_{2} Q \leq a_{1} Q$ [6, Proposition 10.33].

Now if $r_{Q}\left(a_{1}, a_{2}\right) Q a_{2}^{2}=0$ we get $r_{Q}\left(a_{1}, a_{2}\right) \subseteq(1-v) Q$ where $v$ is the central cover of $a_{2}^{2} Q$. As in the previous step this yields

$$
v Q \cong Q /(1-v) Q \leqslant Q / r_{\mathrm{O}}\left(a_{1}, a_{2}\right) .
$$

By the proof of Theorem 9 this implies $v Q \leqslant a_{1} Q \oplus a_{2} Q$. Since $a_{2} Q \leq a_{1} Q$ and $a_{1} Q$ is of type III we thus get $v Q \leq a_{1} Q$ by [6, Corollary 10.17] and once again we are finished.

So we can suppose $r_{Q}\left(a_{1}, a_{2}\right) Q a_{2}^{2} \neq 0$. As before this implies $r_{Q}\left(a_{1}, a_{2}\right) \cap Q a_{2}^{2} Q \neq 0$ and we can find $a_{3} \in r_{Q}\left(a_{1}, a_{2}\right) \cap Q a_{2}^{2} R \cap R$ such that $a_{3}^{2} \neq 0$. If we continue like this then Lemma 7 shows that the process must stop before we reach $a_{n+1}$. Since the process can only stop by yielding a nonzero central idempotent $v \in Q$ such that $v Q \leq a_{1} Q$ we see that $Q_{3}$ is indeed biregular, as required.

There are a couple of interesting special cases of these results. The first, an immediate consequence of letting $n=1$ in Theorems 9 and 11, was proved by Cailleau and Renault in [2, Théorème 2.2] and [15, Proposition 3.7].

COROLlaRY 12. Let $R$ be a reduced ring and $Q$ its maximal right quotient ring. Then $Q=Q_{1} \oplus Q_{3}$ where $Q_{1}$ is a strongly regular self-injective ring and $Q_{3}$ is a biregular right self-injective ring of type III.

COROllary 13. Let $R$ be a prime ring of index $n$ and let $Q$ be its maximal right quotient ring. Then $Q$ is either an $n \times n$ matrix ring over a division ring or a simple directly infinite ring.

Proof. Since $R$ is prime so is $Q$. In particular $Q$ is indecomposable as a ring. By Theorem 9 we see that either $Q$ is of the form $M_{k}(D)$ where $k \leq n$ and $D$ is strongly regular, or $Q$ is of type III. In the former case $D$ is a division ring (since $Q$ is prime) and $k=n$ (since $R \subseteq Q$ has an element of index $n$ ). In the latter case $Q$ is biregular and so simple (being already prime). As $Q$ is type III it is certainly directly infinite and so we are finished. (Alternatively we could deduce this result from Corollary 6 by using [9, Proposition IV.1 and its first corollary] and [8, Theorem 2.5].)

With the help of Corollary 6 we can give a simple criterion for distinguishing the two cases in Corollary 13. Notice that, since it tells us precisely when the maximal right quotient ring of a prime ring $R$ is an $n \times n$ matrix ring over a division ring, the following result may be viewed as a characterization of prime right Goldie rings of dimension $n$ (see [3, p. $76(2)$ and p. $80(7)])$. 
Propostrion 14. Let $R$ be a prime ring of index $n$ and let $Q$ be its maximal right quotient ring. Then $Q$ is an $n \times n$ matrix ring over a division ring if and only if $a R$ is an essential right ideal of $R$ whenever $a \in R$ such that $r_{R}(a)=0$.

Proof. The "only if" part is well known (if $a \in R$ with $r_{R}(a)=0$ then $r_{Q}(a)=0$ and so $a Q \cong Q$; as $Q$ is directly finite $a Q=Q$ and so $a R$ is essential). So suppose that $a R$ is essential whenever $a \in R$ with $r(a)=0$. Since $R$ is prime of index $n$, Corollary 6 say that $R$ is right $S P(m)$ for some $m \leq n$. If $m>1$ then $Q$ is a matrix ring over a division ring (see [8, Theorem 2.3]) and so, by Corollary 13, must be an $n \times n$ matrix ring over a division ring. So suppose $R$ is right $S P(1)$. Hence if $0 \neq x \in R$ there is some $s \in R$ with $r(x s)=0$. By our hypothesis $x s R$, and hence $x R$, is an essential right ideal. Since $R$ is right nonsingular by Proposition 4(c) it follows firstly that $R$ is a domain and then that $R$ is a right Ore domain. Hence $R$ has index 1 and $Q$ is a division ring. Thus the result is proved.

Now let $R$ be any ring of bounded index and let $Q$ be its maximal right quotient ring. The starting point for the above results about $Q$ was the decomposition of $Q$ as a direct product of rings of types I, II and III. This decomposition of $Q$ is in fact available whenever $Q$ is regular and right self-injective, that is, whenever $R$ is right nonsingular. Thus it is natural to ask whether the above results remain true if $R$, instead of being semiprime, is merely right nonsingular. The next result shows that we can no longer say anything special about type I part of $Q$.

PROPOSTTION 15. Every regular right self-injective ring of type $I$ is the maximal right quotient ring of some ring of index at most 2.

Proof. Let $Q$ be a regular right self-injective ring of type I and let $e \in Q$ be a faithful abelian idempotent of $Q$. Thus $r(e Q)=0$ and, by [6, Theorem 3.2], eQe contains no nonzero nilpotent elements. Let $R$ be the subring $Q e$. Writing $R=e Q e+(1-e) Q e$ we see easily that the only nilpotent elements of $R$ lie in $(1-e) Q e$. Thus $R$ has index at most 2 . On the other hand if $0 \neq q \in Q$ we have $q Q e \neq 0$ since $l(Q e)=l(Q e Q)=r(Q e Q)=0$. Since $q Q e \subseteq Q e=R$ we thus have $q R \cap R \neq 0$. Hence $Q$ is a right quotient ring (and so the maximal right quotient ring) of $R$, as desired. (Readers who would prefer that $R$ had an identity could achieve this by using instead $R=Q e+Z$ where $Z$ is the centre of $Q$.)

By choosing $Q$ in the above proof to be an infinite dimensional full linear ring [6, Proposition 10.2] we see in particular that $Q$ can be of type $\mathrm{I}_{\infty}$ (as opposed to the type $\mathrm{I}_{k}$ rings of Theorem 9) and that $Q$ need not be biregular (as $Q$ is prime but not simple [10, p. 93]). Our result about the type III part of $Q$ also breaks down when $R$ is not semiprime, as the following example shows.

ExAmple. Let $S$ be a domain not satisfying the right Ore condition and let $T$ be its maximal right quotient ring. Thus $T$ is a simple regular right self-injective ring of type III. Let $\alpha$ be the smallest cardinal number such that $T_{T} \neq \equiv E\left(\alpha T_{T}\right)$, where $E()$ denotes the injective envelope of a module, and let $Q=\operatorname{End}_{T} E\left(\beta T_{T}\right)$ where $\beta \geq \alpha$. Then $Q$ is a prime 
regular right self-injective ring of type III (see [6, Corollary 9.3 and Proposition 10.12]). Furthermore the discussion on page 157 of [6] shows, in conjunction with [6, Theorem 12.21], that $Q$ has proper ideals. Being prime $Q$ therefore cannot be biregular. However $Q$ is the maximal right quotient ring of a ring of index 2. To see this let $e \in Q$ be a projection from $E\left(\beta T_{\mathrm{T}}\right)$ onto one of the summands $T_{\mathrm{T}}$. Then $e Q e \cong T$ as rings and so $e Q e$ is the maximal right quotient ring of some domain $S^{\prime}$. Putting $R=S^{\prime}+(1-e) Q e$ (and proceeding as in Proposition 15) gives a ring $R$ of index 2 with maximal right quotient ring $Q$.

In fact only one of our results remains true without the semiprimeness of $R$ : the type II part of $Q$ is still zero. To see this we need a final lemma.

LEMma 16. Let $R$ be a ring of index $n$ and let $N$ be the sum of the nilpotent ideals of $R$. If $N$ is essential as a right ideal of $R$ then the right singular ideal $Z_{r}(R)$ is nonzero.

Proof. Choose $a \in R$ such that $a^{n-1} \neq 0$ and $a^{n}=0$. We show that $a^{n-1} \in Z_{r}(R)$. Suppose on the contrary that $r\left(a^{n-1}\right) \cap B=0$ for some nonzero right ideal $B$ of $R$. Since $N$ is essential as a right ideal we may suppose that $B$ is nilpotent and, indeed, that $B^{2}=0$.

Since $R$ has index $n$ we have the following property:

$$
\left.{ }^{*}\right) \ldots \text { if } x \in R \text { with } x^{2}=x a=0 \text { then } a^{n-1} x=0 .
$$

(To see this let $z=x+a$ so that $z^{n}=a^{n-1} x+a^{n}=a^{n-1} x$ and $z^{n+1}=0$.)

Choose $0 \neq b \in B$. Since $r\left(a^{n-1}\right) \cap B=0$ we have $a^{n-1} b \neq 0$. Since $b^{2}=0$ we must, by $\left(^{*}\right)$, have $b a \neq 0$. As $b a \in B$ this implies $a^{n-1}(b a) \neq 0$ and so $b a^{2}=(b a) a \neq 0$ (using (*) again, since $\left.(b a)^{2}=0\right)$. Continuing like this we eventually get $b a^{n-1} \neq 0$. Then $a^{n-1} b a^{n-1} \neq 0$ and a final application of $\left(^{*}\right)$ yields $\left(b a^{n-1}\right) a \neq 0$ which contradicts the fact that $a^{n}=0$. Hence $a^{n-1} \in Z_{r}(R)$ as required.

THEOREM 17. Let $R$ be a right nonsingular ring of bounded index and let $Q$ be its maximal right quotient ring. The type II part of $Q$ is zero.

Proof. Suppose $Q$ has nonzero type II part $Q_{2}$. By working inside $Q_{2}$ (and replacing $R$ with $R \cap Q_{2}$ ) we may assume that $Q$ itself is of type II. Let $N$ be the sum of the nilpotent ideals of $R$. We shall show that $N$ is essential as a right ideal of $R$ and this contradiction of Lemma 16 will complete the proof.

Let $A$ be a nonzero right ideal of $R$ and choose $0 \neq a \in A$. Notice that $A$ meets every nonzero $R$-submodule of $a Q$ nontrivially. As $Q$ is of type II we may suppose that $a Q$ is directly finite and contains no nonzero abelian submodules [6, Proposition 10.8]. By [7, Proposition 5.8] there is some nonzero $a_{1} \in a Q$ such that $(n+1) a_{1} Q \leq a Q$ (where $n$ is the index of $R$ as usual). Since $A \cap a_{1} Q \neq 0$ we may suppose that $a_{1} \in A$. We are going to use Lemma 7 again but this time we must make sure that $a_{1}, a_{2}, \ldots, a_{n+1}$ all lie inside $A$.

We begin the search for $a_{2}$ by showing that $n\left(a_{1} Q\right) \leq a Q \cap r_{Q}\left(a_{1}\right)$. We have the following obvious maps

$$
a Q / a Q \cap r_{Q}\left(a_{1}\right) \cong a Q+r_{Q}\left(a_{1}\right) / r_{Q}\left(a_{1}\right) \leqslant Q / r_{Q}\left(a_{1}\right) \cong a_{1} Q
$$


and so $(n+1) a_{1} Q \leq a Q \leq\left[a Q \cap r_{\mathrm{Q}}\left(a_{1}\right)\right] \oplus a_{1} Q$. Since $a_{1} Q \subseteq a Q$ is directly finite we can use the cancellation property [6, Corollary 9.19] to deduce $n\left(a_{1} Q\right) \leq a Q \cap r_{Q}\left(a_{1}\right)$ as desired. By Lemma 8 (with $X=a Q \cap r_{\mathrm{Q}}\left(a_{1}\right)$ and $q=a_{1}$ ) there is a nonzero $a_{2} \in$ $a Q \cap r_{\mathrm{Q}}\left(a_{1}\right) \cap Q a_{1} R \cap R$ with $a_{2} Q \leq a_{1} Q$. We may suppose that $a_{2} \in A$ (since $a_{2} \in a Q$ anyway). If $a_{2} R$ is a nil right ideal then Proposition 3 gives $\left(a_{2} R\right)^{n} \subseteq N$ which would imply that $A \cap N \neq 0$ (if $\left(a_{2} R\right)^{n} \neq 0$ this is clear and if $\left(a_{2} R\right)^{n}=0$ then $\left.a_{2} R \subseteq N\right)$. In that case we are finished, so we may suppose that $a_{2}^{2} \neq 0$.

We can now continue the construction, showing that $(n-1) a_{1} Q \leq a Q \cap r_{Q}\left(a_{1}, a_{2}\right)$ by means of the embeddings

$$
a Q / a Q \cap r_{\mathrm{Q}}\left(a_{1}, a_{2}\right) \leqslant Q / r_{\mathrm{Q}}\left(a_{1}, a_{2}\right) \leqslant a_{1} Q \oplus a_{2} Q \leqslant 2\left(a_{1} Q\right)
$$

and the cancellation property (as before). In particular $a_{2}^{2} Q \leq a Q \cap r_{Q}\left(a_{1}, a_{2}\right)$ and another application of Lemma 8 then yields either some $a_{3} \in a Q \cap r_{Q}\left(a_{1}, a_{2}\right) \cap Q a_{2}^{2} R \cap R$ with $a_{3}^{2} \neq 0$ or $A \cap N \neq 0$. The process continues until we run out of copies of $a_{1} Q$ to cancel. Either we get $A \cap N \neq 0$ at some stage or we construct elements $a_{1}, a_{2}, \ldots, a_{n+1}$ of $A$ satisfying the hypotheses of Lemma 7 . Since Lemma 7 then gives a nonzero nilpotent right ideal of $R$ inside some $a_{i} R$ we get $A \cap N \neq 0$ anyway. Thus $N$ is indeed essential as a right ideal and we are finished.

\section{REFERENCES}

1. E. P. Armendariz and S. A. Steinberg, Regular self-injective rings with a polynomial identity, Trans. Amer. Math. Soc. 190 (1974), 417-425.

2. A. Cailleau and G. Renault, Sur l'enveloppe injective des anneaux semi-premiers à idéal singulier nul, J. Algebra 15 (1970), 133-141.

3. C. Faith, Lectures on injective modules and quotient rings Lecture Notes in Mathematics No. 49 (Springer-Verlag, 1967).

4. J. W. Fisher, Structure of semiprime P.I. rings, Proc. Amer. Math. Soc. 39 (1973), 465-467.

5. A. W. Goldie, Semiprime rings with maximum condition, Proc. London Math. Soc. (3) 10 (1960), 201-220.

6. K. R. Goodearl, Von Neumann regular rings, (Pitman, 1979).

7. K. R. Goodearl and A. K. Boyle, Dimension theory for nonsingular injective modules, Memoirs Amer. Math. Soc. 177 (1976). 797-834.

8. K. R. Goodearl and D. Handelman, Simple self-injective rings, Comm in Algebra 3 (1975), 209-223.

9. D. Handelman and J. Lawrence, Strongly prime rings, Trans. Amer. Math. Soc. 211 (1975),

10 N. Jacobson, Structure of rings, Amer. Math. Soc. Colloq. Publ. 37 (Providence, R. I., 1964).

11. I. Kaplansky, Topological representation of algebras, II. Trans. Amer. Math. Soc. 68 (1950), 62-75.

12. J. Levitzki, On a problem of A. Kurosch, Bull. Amer. Math. Soc. 52 (1946), 1033-1035.

13. M. Nagata, On the nilpotency of nil algebras, J. Math. Soc. Japan 4 (1952), 296-301.

14. E. C. Posner, Prime rings satisfying a polynomial identity, Proc. Amer. Math. Soc. 11 (1960), 180-183.

15. G. Renault, Anneaux biréguliers auto-injectifs à droite, J. Algebra 36 (1975), 77-84.

16. L. H. Rowen, Monomial conditions on rings, Israel J. Math. 23 (1976), 19-30. 
17. H. Tominaga, Some remarks on $\pi$-regular rings of bounded index, Math. J. Okayama 4 (1954-5), 135-141.

18. Y. Utumi, On quotient rings, Osaka Math. J. 8 (1956), 1-18.

Department of Mathematics

UNIVERSITY OF MELBOURNE

ParkVille, Victoria 3052

Australia
Present address: University College

DUBLIN

IRELAND 